\title{
Convolution Theorems for Quaternion Fourier Transform: Properties and Applications
}

\author{
Mawardi Bahri, ${ }^{1}$ Ryuichi Ashino, ${ }^{2}$ and Rémi Vaillancourt ${ }^{3}$ \\ ${ }^{1}$ Department of Mathematics, Hasanuddin University, Makassar 90245, Indonesia \\ ${ }^{2}$ Division of Mathematical Sciences, Osaka Kyoiku University, Osaka 582-8582, Japan \\ ${ }^{3}$ Department of Mathematics and Statistics, University of Ottawa, Ottawa, ON, Canada K1N 6N5
}

Correspondence should be addressed to Ryuichi Ashino; ashino@cc.osaka-kyoiku.ac.jp

Received 1 June 2013; Revised 1 September 2013; Accepted 7 September 2013

Academic Editor: Narcisa C. Apreutesei

Copyright (C) 2013 Mawardi Bahri et al. This is an open access article distributed under the Creative Commons Attribution License, which permits unrestricted use, distribution, and reproduction in any medium, provided the original work is properly cited.

\begin{abstract}
General convolution theorems for two-dimensional quaternion Fourier transforms (QFTs) are presented. It is shown that these theorems are valid not only for real-valued functions but also for quaternion-valued functions. We describe some useful properties of generalized convolutions and compare them with the convolution theorems of the classical Fourier transform. We finally apply the obtained results to study hypoellipticity and to solve the heat equation in quaternion algebra framework.
\end{abstract}

\section{Introduction}

Convolution is a mathematical operation with several applications in pure and applied mathematics such as numerical analysis, numerical linear algebra, and the design and implementation of finite impulse response filters in signal processing. In [1-3], the authors introduced the Clifford convolution. It is found that some properties of convolution, when generalized to the Clifford Fourier transform (CFT), are very similar to the classical ones.

On the other hand, the quaternion Fourier transform (QFT) is a nontrivial generalization of the classical Fourier transform (FT) using quaternion algebra. The QFT has been shown to be related to the other quaternion signal analysis tools such as quaternion wavelet transform, fractional quaternion Fourier transform, quaternionic windowed Fourier transform, and quaternion Wigner transform [4-9]. A number of already known and useful properties of this extended transform are generalizations of the corresponding properties of the FT with some modifications, but the generalization of convolution theorems of the QFT is still an open problem. In the recent past, several authors [10-13] tried to formulate convolution theorems for the QFT. But they only treated them for real-valued functions which is quite similar to the classical case. In [14], the authors briefly introduced, without proof, the QFT of the convolution of two-dimensional quaternion signals.

In this paper, we establish general convolutions for QFT. Because quaternion multiplication is not commutative, we find new properties of the QFT of convolution of two quaternion-valued functions. These properties describe closely the relationship between the quaternion convolution and its QFT. The generalization of the convolution theorems of the QFT is mainly motivated by the Clifford convolution of general geometric Fourier transform, which has been recently studied in $[15,16]$. We further establish the inverse QFT of the product of the QFT, which is very useful in solving partial differential equations in quaternion algebra framework.

This paper consists of the following sections. Section 2 deals with some results on the real quaternion algebra and the definition of the QFT and its basic properties. We also review some basic properties of QFT, which will be necessary in the next section. Section 3 establishes convolution theorems of QFT and some of their consequences. Section 4 presents an application of QFT to study hypoellipticity and to solve the heat equation in quaternion algebra. Some conclusions are drawn in Section 5. 


\section{Quaternion Algebra}

For convenience, we specify the notation used in this paper. The quaternion algebra over $\mathbb{R}$, denoted by $\mathbb{U}$, is an associative noncommutative four-dimensional algebra,

$$
\mathbb{H}=\left\{q=q_{0}+\mathbf{i} q_{1}+\mathbf{j} q_{2}+\mathbf{k} q_{3} ; q_{0}, q_{1}, q_{2}, q_{3} \in \mathbb{R}\right\},
$$

which obeys the following multiplication rules:

$$
\begin{gathered}
\mathbf{i j}=-\mathbf{j i}=\mathbf{k}, \quad \mathbf{j k}=-\mathbf{k} \mathbf{j}=\mathbf{i}, \quad \mathbf{k} \mathbf{i}=-\mathbf{i k}=\mathbf{j}, \\
\mathbf{i}^{2}=\mathbf{j}^{2}=\mathbf{k}^{2}=\mathbf{i j k}=-1 .
\end{gathered}
$$

For a quaternion $q=q_{0}+\mathbf{i} q_{1}+\mathbf{j} q_{2}+\mathbf{k} q_{3} \in \mathbb{H}, q_{0}$ is called the scalar part of $q$ denoted by $\mathrm{Sc}(q)$ and $\mathbf{i} q_{1}+\mathbf{j} q_{2}+\mathbf{k} q_{3}$ is called the vector (or pure) part of $q$. The vector part of $q$ is conventionally denoted by $\mathbf{q}$. Let $p, q \in \mathbb{H}$ and let $\mathbf{p}, \mathbf{q}$ be their vector parts, respectively. It is common to write for short

$$
\begin{gathered}
\mathbf{q} \cdot \mathbf{p}=q_{1} p_{1}+q_{2} p_{2}+q_{3} p_{3}, \\
\mathbf{q} \times \mathbf{p}=\mathbf{i}\left(q_{2} p_{3}-q_{3} p_{2}\right)+\mathbf{j}\left(q_{3} p_{1}-q_{1} p_{3}\right)+\mathbf{k}\left(q_{1} p_{2}-q_{2} p_{1}\right) .
\end{gathered}
$$

Then, (2) yields the quaternionic multiplication $q p$ as

$$
\mathrm{q} p=q_{0} p_{0}-\mathbf{q} \cdot \mathbf{p}+q_{0} \mathbf{p}+p_{0} \mathbf{q}+\mathbf{q} \times \mathbf{p} .
$$

The quaternion conjugate of $q$, given by

$$
\bar{q}=q_{0}-\mathbf{i} q_{1}-\mathbf{j} q_{2}-\mathbf{k} q_{3}, \quad q_{0}, q_{1}, q_{2}, q_{3} \in \mathbb{R},
$$

is an anti-involution; that is,

$$
\overline{q p}=\bar{p} \bar{q} .
$$

From (5) we obtain the norm or modulus of $q \in \mathbb{U}$ defined as

$$
|q|=\sqrt{q \bar{q}}=\sqrt{q_{0}^{2}+q_{1}^{2}+q_{2}^{2}+q_{3}^{2}} .
$$

It is not difficult to see that

$$
|q p|=|q||p|, \quad \forall p, q \in \mathbb{H} .
$$

Using the conjugate (5) and the modulus of $q$, we can define the inverse of $q \in \mathbb{U} \backslash\{0\}$ as

$$
q^{-1}=\frac{\bar{q}}{|q|^{2}}
$$

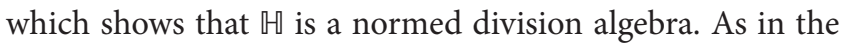
algebra of complex numbers, we can define three nontrivial quaternion involutions [10]:

$$
\begin{aligned}
& \alpha(q)=-\mathbf{i} q \mathbf{i}=-\mathbf{i}\left(q_{0}+\mathbf{i} q_{1}+\mathbf{j} q_{2}+\mathbf{k} q_{3}\right) \mathbf{i} \\
& =q_{0}+\mathbf{i} q_{1}-\mathbf{j} q_{2}-\mathbf{k} q_{3}, \\
& \beta(q)=-\mathbf{j} q \mathbf{j}=-\mathbf{j}\left(q_{0}+\mathbf{i} q_{1}+\mathbf{j} q_{2}+\mathbf{k} q_{3}\right) \mathbf{j} \\
& =q_{0}-\mathbf{i} q_{1}+\mathbf{j} q_{2}-\mathbf{k} q_{3}, \\
& \gamma(q)=-\mathbf{k} q \mathbf{k}=-\mathbf{k}\left(q_{0}+\mathbf{i} q_{1}+\mathbf{j} q_{2}+\mathbf{k} q_{3}\right) \mathbf{k} \\
& =q_{0}-\mathbf{i} q_{1}-\mathbf{j} q_{2}+\mathbf{k} q_{3} .
\end{aligned}
$$

Hereinafter, besides the quaternion units $\mathbf{i}, \mathbf{j}$, and $\mathbf{k}$ and the vector part $\mathbf{q}$ of a quaternion $q \in \mathbb{H}$, we will use the real vector notation:

$$
\begin{aligned}
\mathbf{x}=\left(x_{1}, x_{2}\right) \in \mathbb{R}^{2}, & |\mathbf{x}|^{2}=x_{1}^{2}+x_{2}^{2}, \\
\mathbf{x} \cdot \mathbf{y}=x_{1} y_{1}+x_{2} y_{2}, & f(\mathbf{x})=f\left(x_{1}, x_{2}\right),
\end{aligned}
$$

and so on when there is no confusion. This gives the following definition.

Definition 1 (see [10]). A function $f: \mathbb{R}^{2} \rightarrow \mathbb{H}$ is called quaternionic Hermitian if, for the involutions $\alpha$ and $\beta$,

$$
f\left(-x_{1}, x_{2}\right)=\beta(f(\mathbf{x})), \quad f\left(x_{1},-x_{2}\right)=\alpha(f(\mathbf{x})),
$$

for each $\mathbf{x} \in \mathbb{R}^{2}$.

For any unit quaternion

$$
q=q_{0}+\mathbf{q}=\cos \left(\frac{\theta}{2}\right)+\sin \left(\frac{\theta}{2}\right)
$$

and for any vector $\mathbf{v} \in \mathbb{R}^{3}$ the action of the operator

$$
L_{q}(\mathbf{v})=q \mathbf{v} \bar{q}
$$

on $\mathbf{v}$ is equivalent to a rotation of the vector $\mathbf{v}$ through an angle $\theta$ about $\mathbf{u}$ as the axis of rotation.

It is convenient to introduce an inner product for two functions $f, g: \mathbb{R}^{2} \rightarrow \mathbb{H}$ as follows:

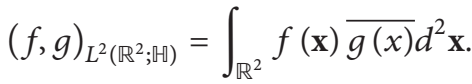

In particular, for $f=g$, we obtain the scalar product of the above inner product (15) given by

$$
\langle f, f\rangle=\|f\|_{L^{2}\left(\mathbb{R}^{2} ; \mathbb{W}\right)}=\left(\int_{\mathbb{R}^{2}}|f(x)|^{2} d^{2} x\right)^{1 / 2} .
$$

2.1. Multiindices and Derivatives. A couple $\boldsymbol{\alpha}=\left(\alpha_{1}, \alpha_{2}\right)$ of nonnegative integers is called a multiindex. We denote

$$
|\boldsymbol{\alpha}|=\alpha_{1}+\alpha_{2}, \quad \boldsymbol{\alpha} !=\alpha_{1} ! \alpha_{2} !
$$

and for $\mathbf{x} \in \mathbb{R}^{2}$,

$$
\mathbf{x}^{\alpha}=x_{1}^{\alpha_{1}} x_{2}^{\alpha_{2}}
$$

Derivatives are conveniently expressed by multiindices:

$$
\partial^{\boldsymbol{\alpha}}=\frac{\partial^{|\boldsymbol{\alpha}|}}{\partial x_{1}^{\alpha_{1}} \partial x_{2}^{\alpha_{2}}} .
$$

Denote by $\left\{\mathbf{e}_{1}, \mathbf{e}_{2}\right\}$ the standard basis of $\mathbb{R}^{2}$. The vector differential $\mathbf{a} \cdot \nabla$ along the direction $\mathbf{a}$ is defined by

$$
\mathbf{a} \cdot \nabla=a_{1} \partial_{1}+a_{2} \partial_{2}
$$

where $\nabla=\mathbf{e}_{1} \partial_{1}+\mathbf{e}_{2} \partial_{2}$. 


\subsection{QFT and Its Properties}

Definition 2. The QFT of $f \in L^{2}\left(\mathbb{R}^{2} ; \mathbb{H}\right)$ is the transform $\mathscr{F}_{q}\{f\} \in L^{2}\left(\mathbb{R}^{2}, \mathbb{M}\right)$ given by the integral

$$
\mathscr{F}_{q}\{f\}(\boldsymbol{\omega})=\int_{\mathbb{R}^{2}} e^{-\mathbf{i} \omega_{1} x_{1}} f(\mathbf{x}) e^{-\mathbf{j} \omega_{2} x_{2}} d \mathbf{x},
$$

where $\mathscr{F}_{q}$ is called the quaternion Fourier transform operator or the quaternion Fourier transformation.

Using the Euler formula for the quaternion Fourier kernel $e^{-\mathbf{i} \omega_{1} x_{1}} e^{-\mathbf{j} \omega_{2} x_{2}}$, we can rewrite (21) in the following form:

$$
\begin{aligned}
\mathscr{F}_{q}\{f\}(\boldsymbol{\omega})= & \int_{\mathbb{R}^{2}} f(\mathbf{x}) \cos \left(\omega_{1} x_{1}\right) \cos \left(\omega_{2} x_{2}\right) d \mathbf{x} \\
& -\int_{\mathbb{R}^{2}} \mathbf{i} f(\mathbf{x}) \sin \left(\omega_{1} x_{1}\right) \cos \left(\omega_{2} x_{2}\right) d \mathbf{x} \\
& -\int_{\mathbb{R}^{2}} f(\mathbf{x}) \mathbf{j} \cos \left(\omega_{1} x_{1}\right) \sin \left(\omega_{2} x_{2}\right) d \mathbf{x} \\
& +\int_{\mathbb{R}^{2}} \mathbf{i} f(\mathbf{x}) \mathbf{j} \sin \left(\omega_{1} x_{1}\right) \sin \left(\omega_{2} x_{2}\right) d \mathbf{x} .
\end{aligned}
$$

Definition 3. The inverse QFT of $g \in L^{2}\left(\mathbb{R}^{2} ; \mathbb{M}\right)$ is the transform $\mathscr{F}_{q}^{-1}\{g\} \in L^{2}\left(\mathbb{R}^{2}, \mathbb{H}\right)$ given by the integral

$$
\mathscr{F}_{q}^{-1}[g(\boldsymbol{\omega})](\mathbf{x})=\frac{1}{(2 \pi)^{2}} \int_{\mathbb{R}^{2}} e^{\mathrm{i} \omega_{1} x_{1}} g(\boldsymbol{\omega}) e^{\mathrm{j} \omega_{2} x_{2}} d \boldsymbol{\omega} .
$$

Some important properties of the QFT are stated in the following lemmas proved in [12, 17].

Lemma 4. Let $f \in L^{1}\left(\mathbb{R}^{2} ; \mathbb{H}\right) \cap L^{2}\left(\mathbb{R}^{2} ; \mathbb{H}\right)$. If $\mathscr{F}_{q}\left\{\partial^{\boldsymbol{\alpha}} f\right\} \in$ $L^{1}\left(\mathbb{R}^{2} ; \mathbb{H}\right)$, then

$$
\mathscr{F}_{q}\left\{\partial^{\boldsymbol{\alpha}} f\right\}(\boldsymbol{\omega})=\left(\mathbf{i} \omega_{1}\right)^{\alpha_{1}} \mathscr{F}_{q}\{f\}(\boldsymbol{\omega})\left(\mathbf{j} \omega_{2}\right)^{\alpha_{2}} .
$$

In particular, if $\mathscr{F}_{q}\left\{\partial^{(2,0)} f\right\} \in L^{1}\left(\mathbb{R}^{2} ; \mathbb{H}\right)$, then

$$
\mathscr{F}_{q}\left\{\partial^{(2,0)} f\right\}(\boldsymbol{\omega})=\left(\mathbf{i} \omega_{1}\right)^{2} \mathscr{F}_{q}\{f\}(\boldsymbol{\omega}) .
$$

And if $\mathscr{F}_{q}\left\{\partial^{(0,2)} f\right\} \in L^{1}\left(\mathbb{R}^{2} ; \mathbb{H}\right)$, then

$$
\mathscr{F}_{q}\left\{\partial^{(0,2)} f\right\}(\boldsymbol{\omega})=\mathscr{F}_{q}\{f\}(\boldsymbol{\omega})\left(\mathbf{j} \omega_{2}\right)^{2} .
$$

Lemma 5 (scalar QFT Parseval). The scalar product of $f, g \in$ $L^{2}\left(\mathbb{R}^{2} ; \mathbb{H}\right)$ and its QFT are related by

$$
\langle f, g\rangle_{L^{2}\left(\mathbb{R}^{2} ; \mathbb{G}\right)}=\frac{1}{(2 \pi)^{2}}\left\langle\mathscr{F}_{q}\{f\}, \mathscr{F}_{q}\{g\}\right\rangle_{L^{2}\left(\mathbb{R}^{2} ; \mathbb{H}\right)} .
$$

And in particular, with $f=g$, the Plancherel theorem indicates that

$$
\|f\|_{L^{2}\left(\mathbb{R}^{2} ; \mathbb{W}\right)}^{2}=\frac{1}{(2 \pi)^{2}}\left\|\mathscr{F}_{q}\{f\}\right\|_{L^{2}\left(\mathbb{R}^{2} ; \mathbb{W}\right)}^{2} .
$$

This shows that the total signal energy computed in the spatial domain is equal to the total signal energy computed in the quaternion domain.

\section{Convolution of QFT}

In this section, we establish the quaternion convolution of the QFT which extends the classical convolution to quaternion fields. Let us first define the convolution of two quaternionvalued functions.

Definition 6. The convolution of $f \in L^{2}\left(\mathbb{R}^{2} ; \mathbb{U}\right)$ and $g \in$ $L^{2}\left(\mathbb{R}^{2} ; \mathbb{H}\right)$, denoted by $f \star g$, is defined by

$$
(f \star g)(\mathbf{x})=\int_{\mathbb{R}^{2}} f(\mathbf{y}) g(\mathbf{x}-\mathbf{y}) d \mathbf{y} .
$$

Example 7. To illustrate the general noncommutativity ( $f$ * $g) \neq(g \star f)$, let us compute the convolution of $f(\mathbf{x})=\mathbf{k} e^{-|\mathbf{x}|^{2}}$ and $g(\mathbf{x})=(\mathbf{i}+\mathbf{j}) e^{\mathbf{i} \omega_{1} x_{1}} e^{\mathbf{j} \omega_{2} x_{2}}$. Although $g \in L^{\infty}\left(\mathbb{R}^{2} ; \mathbb{U}\right)$, we can still define the convolution of $f$ and $g$, because $f$ decays rapidly at infinity. A simple calculation gives

$$
\begin{aligned}
(f \star g)(\mathbf{x}) & =\int_{\mathbb{R}^{2}} \mathbf{k} e^{-|\mathbf{y}|^{2}}(\mathbf{i}+\mathbf{j}) e^{\mathbf{i} \omega_{1}\left(x_{1}-y_{1}\right)} e^{\mathbf{j} \omega_{2}\left(x_{2}-y_{2}\right)} d \mathbf{y} \\
& =\int_{\mathbb{R}^{2}} \mathbf{k}(\mathbf{i}+\mathbf{j}) e^{\mathbf{i} \omega_{1} x_{1}} e^{-\mathbf{i} \omega_{1} y_{1}} e^{-|\mathbf{y}|^{2}} e^{-\mathbf{j} \omega_{2} y_{2}} d \mathbf{y} e^{\mathbf{j} \omega_{2} x_{2}} \\
& =\mathbf{k}(\mathbf{i}+\mathbf{j}) e^{\mathbf{i} \omega_{1} x_{1}} e^{-|\boldsymbol{\omega}|^{2} / 4} e^{\mathbf{j} \omega_{2} x_{2}} \\
& =(\mathbf{j}-\mathbf{i}) e^{\mathbf{i} \omega_{1} x_{1}} e^{-|\omega|^{2} / 4} e^{\mathbf{j} \omega_{2} x_{2}} .
\end{aligned}
$$

On the other hand, we have

$$
\begin{aligned}
(g * f)(\mathbf{x}) & =\int_{\mathbb{R}^{2}}(\mathbf{i}+\mathbf{j}) e^{\mathbf{i} \omega_{1} y_{1}} e^{\mathbf{j} \omega_{2} y_{2}} \mathbf{k} e^{-|\mathbf{x}-\mathbf{y}|^{2}} d \mathbf{y} \\
& =\int_{\mathbb{R}^{2}}(\mathbf{i}+\mathbf{j}) \mathbf{k} e^{-\mathbf{i} \omega_{1} y_{1}} e^{-|\mathbf{x}-\mathbf{y}|^{2}} e^{-\mathbf{j} \omega_{2} y_{2}} d \mathbf{y} \\
& =\int_{\mathbb{R}^{2}}(\mathbf{i}+\mathbf{j}) \mathbf{k} e^{-\mathbf{i} \omega_{1} y_{1}} e^{-|\mathbf{y}-\mathbf{x}|^{2}} e^{-\mathbf{j} \omega_{2} y_{2}} d \mathbf{y} \\
& =(\mathbf{i}-\mathbf{j}) e^{-\mathbf{i} \omega_{1} x_{1}} e^{-|\boldsymbol{\omega}|^{2} / 4} e^{-\mathbf{j} \omega_{2} x_{2}} .
\end{aligned}
$$

In the following, we summarize the elementary properties of quaternion convolution as shown in Table 1 (compared to Folland [18]).

Lemma 8 (linearity). For quaternion functions $f, g$, and h and quaternion constants $\kappa_{1}$ and $\kappa_{2}$ one gets

$$
\left(\kappa_{1} f+\kappa_{2} g\right) \star h=\kappa_{1}(f \star h)+\kappa_{2}(g \star h) .
$$

One also gets for real constants $\kappa_{1}$ and $\kappa_{2}$ (due to the noncommutativity of the quaternion multiplication, (33) does not hold for quaternion constants $\kappa_{1}$ and $\kappa_{2}$ )

$$
h \star\left(\kappa_{1} f+\kappa_{2} g\right)=\kappa_{1}(h \star f)+\kappa_{2}(h \star g) .
$$

Lemma 9 (shifting). Given a quaternion function $f \in$ $L^{2}\left(\mathbb{R}^{2} ; \mathbb{H}\right)$, let $\tau_{\mathbf{a}} f(\mathbf{x})$ denote the shifted (translated) function defined by $\tau_{\mathbf{a}} f(\mathbf{x})=f(\mathbf{x}-\mathbf{a})$, where $\mathbf{a} \in \mathbb{R}^{2}$. Then one gets

$$
\begin{aligned}
& \left(\tau_{\mathbf{a}} f \star g\right)(\mathbf{x})=\tau_{\mathbf{a}}(f \star g)(\mathbf{x}), \\
& \left(f \star \tau_{\mathbf{a}} g\right)(\mathbf{x})=\tau_{\mathbf{a}}(f \star g)(\mathbf{x}) .
\end{aligned}
$$


TABLE 1: Basic properties of quaternion convolution.

\begin{tabular}{ll}
\hline $\begin{array}{l}\text { Basic } \\
\text { property }\end{array}$ & Quaternion convolution \\
\hline Linearity & $\left(\kappa_{1} f+\kappa_{2} g\right) \star h=\kappa_{1}(f \star h)+\kappa_{2}(f \star h), \quad \kappa_{1}, \kappa_{2} \in \mathbb{H}$, \\
& $h \star\left(\kappa_{1} f+\kappa_{2} g\right)=\kappa_{1}(h \star f)+\kappa_{2}(h \star g), \quad \kappa_{1}, \kappa_{2} \in \mathbb{R}$ \\
Shifting & $\left(\tau_{\mathbf{a}} f \star g\right)=\tau_{\mathbf{a}}(f \star g)$, \\
& $\left(f \star \tau_{\mathbf{a}} g\right)=\tau_{\mathbf{a}}(f \star g)$ \\
Conjugation & $\overline{(f \star g)}=(\bar{g} \star \bar{f})$ \\
Associativity & $(f \star g) \star h=f \star(g \star h)$ \\
Distributivity & $f \star(g+h)=(f \star g)+(f \star h)$ \\
Vector & $\mathbf{a} \cdot \nabla(f \star g)=(\mathbf{a} \cdot \nabla f) \star g=f \star(\mathbf{a} \cdot \nabla g)$ \\
differential & \\
Impulse & $f \star \delta=f$ \\
convolution & $f \star$
\end{tabular}

Proof. For (34), a direct calculation gives

$$
\begin{aligned}
\left(\tau_{\mathbf{a}} f \star g\right)(\mathbf{x}) & =\int_{\mathbb{R}^{2}} \tau_{\mathbf{a}} f(\mathbf{y}) g(\mathbf{x}-\mathbf{y}) d \mathbf{y} \\
& =\int_{\mathbb{R}^{2}} f(\mathbf{y}-\mathbf{a}) g(\mathbf{x}-\mathbf{y}) d \mathbf{y} \\
& =\int_{\mathbb{R}^{2}} f(\mathbf{y}-\mathbf{a}) g((\mathbf{x}-\mathbf{a})-(\mathbf{y}-\mathbf{a})) d \mathbf{y} \\
& =\int_{\mathbb{R}^{2}} f(\mathbf{s}) g((\mathbf{x}-\mathbf{a})-\mathbf{s}) d \mathbf{s} \\
& =(f \star g)(\mathbf{x}-\mathbf{a}) \\
& =\tau_{\mathbf{a}}(f \star g)(\mathbf{x}),
\end{aligned}
$$

which finishes the proof.

Remark 10. From (34) and (35), it is not difficult to see that $\left(\tau_{\mathbf{a}} f \star g\right)(\mathbf{x})=\left(f \star \tau_{\mathrm{a}} g\right)(\mathbf{x})$ and $\left(\tau_{\mathbf{a}} g \star f\right)(\mathbf{x})=\left(g \star \tau_{\mathbf{a}} f\right)(\mathbf{x})$.

Lemma 11 (conjugation). For all quaternion functions $f, g \in$ $L^{2}\left(\mathbb{R}^{2} ; \mathbb{W}\right)$ one has

$$
\overline{(f \star g)}(\mathbf{x})=(\bar{g} \star \bar{f})(x) .
$$

Proof. A straightforward computation gives

$$
\begin{aligned}
\overline{(f \star g)}(\mathbf{x}) & =\int_{\mathbb{R}^{2}} \overline{f(\mathbf{y}) g(\mathbf{x}-\mathbf{y})} d \mathbf{y} \\
& =\int_{\mathbb{R}^{2}} \bar{g}((\mathbf{x}-\mathbf{y})) \bar{f}(\mathbf{y}) d \mathbf{y} \\
& =\int_{\mathbb{R}^{2}} \bar{g}(\mathbf{z}) \bar{f}((\mathbf{x}-\mathbf{z})) d \mathbf{z} \\
& =(\bar{g} \star \bar{f})(\mathbf{x}) .
\end{aligned}
$$

This finishes the proof.
Lemma 12 (see $[2,3]$; vector differential). For all quaternion functions $f, g \in L^{2}\left(\mathbb{R}^{2} ; \mathbb{U}\right)$ one has

$$
\mathbf{a} \cdot \nabla(f \star g)=(\mathbf{a} \cdot \nabla f) \star g=f \star(\mathbf{a} \cdot \nabla g) .
$$

Ell and Sangwine [19] distinguish between right and left discrete quaternion convolution due to the non-commutative property of the quaternion multiplication. Here, we only consider one kind of quaternion convolutions. We come now to the main theorem (generalization of the QFT of the quaternion convolution in general geometric Fourier transform is investigated in [15]. It can easily be seen that the result is closely related to equation (4.30) of [15]) of this paper. This theorem describes the relationship between the convolution of two quaternion functions and its QFT.

Theorem 13. Let $f(\mathbf{x})=f_{0}(\mathbf{x})+\mathbf{i} f_{1}(\mathbf{x})+\mathbf{j} f_{2}(\mathbf{x})+\mathbf{k} f_{3}(\mathbf{x})$ and $g(\mathbf{x})=g_{0}(\mathbf{x})+\mathbf{i} g_{1}(\mathbf{x})+\mathbf{j} g_{2}(\mathbf{x})+\mathbf{k} g_{3}(\mathbf{x})$ be two quaternionvalued functions, then the QFT of the convolution of $f \in$ $L^{2}\left(\mathbb{R}^{2} ; \mathbb{H}\right)$ and $g \in L^{2}\left(\mathbb{R}^{2} ; \mathbb{H}\right)$ is given by

$$
\begin{aligned}
\mathscr{F}_{q}\{f & \star g\}(\boldsymbol{\omega}) \\
= & \left(\mathscr{F}_{q}\left\{f_{0}\right\}(\boldsymbol{\omega})+\mathbf{i} \mathscr{F}_{q}\left\{f_{1}\right\}(\boldsymbol{\omega})\right) \\
& \times\left(\mathscr{F}_{q}\left\{g_{0}\right\}(\boldsymbol{\omega})+\mathbf{j} \mathscr{F}_{q}\left\{g_{2}\right\}\left(-\omega_{1}, \omega_{2}\right)\right) \\
& +\left(\mathscr{F}_{q}\left\{f_{0}\right\}\left(\omega_{1},-\omega_{2}\right)+\mathbf{i} \mathscr{F}_{q}\left\{f_{1}\right\}\left(\omega_{1},-\omega_{2}\right)\right) \\
& \times\left(\mathbf{i} \mathscr{F}_{q}\left\{g_{1}\right\}(\boldsymbol{\omega})+\mathbf{k} \mathscr{F}_{q}\left\{g_{3}\right\}\left(-\omega_{1}, \omega_{2}\right)\right) \\
& +\left(\mathbf{j} \mathscr{F}_{q}\left\{f_{2}\right\}\left(-\omega_{1}, \omega_{2}\right)+\mathbf{k} \mathscr{F}_{q}\left\{f_{3}\right\}\left(-\omega_{1}, \omega_{2}\right)\right) \\
& \times\left(\mathscr{F}_{q}\left\{g_{0}\right\}\left(-\omega_{1}, \omega_{2}\right)+\mathbf{j} \mathscr{F}_{q}\left\{g_{2}\right\}(\boldsymbol{\omega})\right) \\
& +\left(\mathbf{j} \mathscr{F}_{q}\left\{f_{2}\right\}(-\boldsymbol{\omega})+\mathbf{k} \mathscr{F}_{q}\left\{f_{3}\right\}(-\boldsymbol{\omega})\right) \\
& \times\left(\mathbf{i} \mathscr{F}_{q}\left\{g_{1}\right\}\left(-\omega_{1}, \omega_{2}\right)+\mathbf{k} \mathscr{F}_{q}\left\{g_{3}\right\}(\boldsymbol{\omega})\right) .
\end{aligned}
$$

Proof. In this proof we will use the decomposition of quaternion functions and their QFTs. Let $\mathscr{F}_{q}\{f\}(\boldsymbol{\omega})$ and $\mathscr{F}_{q}\{g\}(\boldsymbol{\omega})$ denote the QFT of $f \in L^{2}\left(\mathbb{R}^{2} ; \mathbb{H}\right)$ and $g \in L^{2}\left(\mathbb{R}^{2} ; \mathbb{H}\right)$, respectively. Expanding the QFT of the left-hand side of (40), we immediately get

$$
\begin{aligned}
& \stackrel{\mathscr{F}_{q}}{ }\{f \star g\}(\boldsymbol{\omega}) \\
& \stackrel{(21)}{=} \int_{\mathbb{R}^{2}} e^{-\mathbf{i} \omega_{1} x_{1}}(f \star g)(\mathbf{x}) e^{-\mathbf{j} \omega_{2} x_{2}} d \mathbf{x} \\
& \stackrel{(29)}{=} \int_{\mathbb{R}^{2}} e^{-\mathbf{i} \omega_{1} x_{1}}\left[\int_{\mathbb{R}^{2}} f(\mathbf{y}) g(\mathbf{x}-\mathbf{y}) d \mathbf{y}\right] e^{-\mathbf{j} \omega_{2} x_{2}} d \mathbf{x} \\
& =\int_{\mathbb{R}^{2}} e^{-\mathbf{i} \omega_{1} x_{1}} f(\mathbf{y})\left[\int_{\mathbb{R}^{2}} g(\mathbf{x}-\mathbf{y}) e^{-\mathbf{j} \omega_{2} x_{2}} d \mathbf{x}\right] d \mathbf{y} .
\end{aligned}
$$


By the change of variables $\mathbf{z}=\mathbf{x}-\mathbf{y}$, the above transform can be written as

$$
\begin{aligned}
& \mathscr{F}_{q}\{f \star g\}(\boldsymbol{\omega}) \\
& =\iint_{\mathbb{R}^{2}} e^{-\mathbf{i} \omega_{1}\left(y_{1}+z_{1}\right)} f(\mathbf{y}) g(\mathbf{z}) e^{-\mathbf{j} \omega_{2}\left(y_{2}+z_{2}\right)} d \mathbf{z} d \mathbf{y} \\
& =\int_{\mathbb{R}^{2}} e^{-\mathbf{i} \omega_{1}\left(y_{1}+z_{1}\right)}\left(\left\{f_{0}(\mathbf{y})+\mathbf{i} f_{1}(\mathbf{y})\right\}+\mathbf{j} f_{2}(\mathbf{y})+\mathbf{k} f_{3}(\mathbf{y})\right) \\
& \times \int_{\mathbb{R}^{2}}\left(\left\{g_{0}(\mathbf{z})+\mathbf{j} g_{2}(\mathbf{z})\right\}+\mathbf{i} g_{1}(\mathbf{z})\right. \\
& \left.+\mathbf{k} g_{3}(\mathbf{z})\right) g(\mathbf{z}) e^{-\mathbf{j} \omega_{2}\left(y_{2}+z_{2}\right)} d \mathbf{z} d \mathbf{y} \\
& =\iint_{\mathbb{R}^{2}} e^{-\mathbf{i} \omega_{1}\left(y_{1}+z_{1}\right)}\left(f_{0}(\mathbf{y})+\mathbf{i} f_{1}(\mathbf{y})\right) \\
& \times\left(g_{0}(\mathbf{z})+\mathbf{j} g_{2}(\mathbf{z})\right) e^{-\mathbf{j} \omega_{2}\left(y_{2}+z_{2}\right)} d \mathbf{z} d \mathbf{y} \\
& +\iint_{\mathbb{R}^{2}} e^{-\mathbf{i} \omega_{1}\left(y_{1}+z_{1}\right)}\left(f_{0}(\mathbf{y})+\mathbf{i} f_{1}(\mathbf{y})\right) \\
& \times\left(\mathbf{i} g_{1}(\mathbf{z})+\mathbf{k} g_{3}(\mathbf{z})\right) e^{-\mathbf{j} \omega_{2}\left(y_{2}+z_{2}\right)} d \mathbf{z} d \mathbf{y} \\
& +\iint_{\mathbb{R}^{2}} e^{-\mathbf{i} \omega_{1}\left(y_{1}+z_{1}\right)}\left(\mathbf{j} f_{2}(\mathbf{y})+\mathbf{k} f_{3}(\mathbf{y})\right) \\
& \times\left(g_{0}(\mathbf{z})+\mathbf{j} g_{2}(\mathbf{z})\right) e^{-\mathbf{j} \omega_{2}\left(y_{2}+z_{2}\right)} d \mathbf{z} d \mathbf{y} \\
& +\iint_{\mathbb{R}^{2}} e^{-\mathbf{i} \omega_{1}\left(y_{1}+z_{1}\right)}\left(\mathbf{j} f_{2}(\mathbf{y})+\mathbf{k} f_{3}(\mathbf{y})\right) \\
& \times\left(\mathbf{i} g_{1}(\mathbf{z})+\mathbf{k} g_{3}(\mathbf{z})\right) e^{-\mathbf{j} \omega_{2}\left(y_{2}+z_{2}\right)} d \mathbf{z} d \mathbf{y} \\
& =\iint_{\mathbb{R}^{2}} e^{-\mathbf{i} \omega_{1} y_{1}}\left(f_{0}(\mathbf{y})+\mathbf{i} f_{1}(\mathbf{y})\right) \\
& \times\left(\mathscr{F}_{q}\left\{g_{0}\right\}(\boldsymbol{\omega})+\mathbf{j} \mathscr{F}_{q}\left\{g_{2}\right\}\left(-\omega_{1}, \omega_{2}\right)\right) \\
& \times e^{-\mathbf{j} \omega_{2} y_{2}} d \mathbf{z} d \mathbf{y} \\
& +\iint_{\mathbb{R}^{2}} e^{-\mathbf{i} \omega_{1} y_{1}}\left(f_{0}(\mathbf{y})+\mathbf{i} f_{1}(\mathbf{y})\right) \\
& \times\left(\mathbf{i} \mathscr{F}_{q}\left\{g_{1}\right\}(\boldsymbol{\omega})+\mathbf{k} \mathscr{F}_{q}\left\{g_{3}\right\}\left(-\omega_{1}, \omega_{2}\right)\right) \\
& \times e^{-\mathbf{j} \omega_{2} y_{2}} d \mathbf{z} d \mathbf{y} \\
& +\iint_{\mathbb{R}^{2}} e^{-\mathbf{i} \omega_{1} y_{1}}\left(\mathbf{j} f_{2}(\mathbf{y})+\mathbf{k} f_{3}(\mathbf{y})\right) \\
& \times\left(\mathscr{F}_{q}\left\{g_{0}\right\}\left(-\omega_{1}, \omega_{2}\right)+\mathbf{j} \mathscr{F}_{q}\left\{g_{2}\right\}(\boldsymbol{\omega})\right) \\
& \times e^{-\mathbf{j} \omega_{2} y_{2}} d \mathbf{z} d \mathbf{y} \\
& +\iint_{\mathbb{R}^{2}} e^{-\mathbf{i} \omega_{1} y_{1}}\left(\mathbf{j} f_{2}(\mathbf{y})+\mathbf{k} f_{3}(\mathbf{y})\right) \\
& \times\left(\mathbf{i} \mathscr{F}_{q}\left\{g_{1}\right\}\left(-\omega_{1}, \omega_{2}\right)+\mathbf{k} \mathscr{F}_{q}\left\{g_{3}\right\}(\boldsymbol{\omega})\right) \\
& \times e^{-\mathbf{j} \omega_{2} y_{2}} d \mathbf{z} d \mathbf{y}
\end{aligned}
$$

$$
\begin{aligned}
=\int_{\mathbb{R}^{2}} e^{-\mathbf{i} \omega_{1} y_{1}}\left(f_{0}(\mathbf{y})+\mathbf{i} f_{1}(\mathbf{y})\right) e^{-\mathbf{j} \omega_{2} y_{2}} d \mathbf{y} \\
\quad \times\left(\mathscr{F}_{q}\left\{g_{0}\right\}(\boldsymbol{\omega})+\mathbf{j} \mathscr{F}_{q}\left\{g_{2}\right\}\left(-\omega_{1}, \omega_{2}\right)\right) \\
+\int_{\mathbb{R}^{2}} e^{-\mathbf{i} \omega_{1} y_{1}}\left(f_{0}(\mathbf{y})+\mathbf{i} f_{1}(\mathbf{y})\right) e^{\mathbf{j} \omega_{2} y_{2}} d \mathbf{y} \\
\quad \times\left(\mathbf{i} \mathscr{F}_{q}\left\{g_{1}\right\}(\boldsymbol{\omega})+\mathbf{k} \mathscr{F}_{q}\left\{g_{3}\right\}\left(-\omega_{1}, \omega_{2}\right)\right) \\
+\int_{\mathbb{R}^{2}} e^{-\mathbf{i} \omega_{1} y_{1}}\left(\mathbf{j} f_{2}(\mathbf{y})+\mathbf{k} f_{3}(\mathbf{y})\right) e^{-\mathbf{j} \omega_{2} y_{2}} d \mathbf{y} \\
\quad \times\left(\mathscr{F}_{q}\left\{g_{0}\right\}\left(-\omega_{1}, \omega_{2}\right)+\mathbf{j} \mathscr{F}_{q}\left\{g_{2}\right\}(\boldsymbol{\omega})\right) \\
+\int_{\mathbb{R}^{2}} e^{-i \omega_{1} y_{1}}\left(\mathbf{j} f_{2}(y)+\mathbf{k} f_{3}(\mathbf{y})\right) e^{\mathbf{j} \omega_{2} y_{2}} d \mathbf{y} \\
\quad \times\left(\mathbf{i} \mathscr{F}_{q}\left\{g_{1}\right\}\left(-\omega_{1}, \omega_{2}\right)+\mathbf{k} \mathscr{F}_{q}\left\{g_{3}\right\}(\boldsymbol{\omega})\right),
\end{aligned}
$$

where the assumption $\mathscr{F}_{q}\left\{g_{i}\right\} \in L^{2}\left(\mathbb{R}^{2} ; \mathbb{R}\right)$ for $i=1,2,3$ is used in the fourth line. This gives the desired result.

The following lemmas are special cases of Theorem 13.

Lemma 14. Let $f, g \in L^{2}\left(\mathbb{R}^{2} ; \mathbb{U}\right)$, where

$$
f=f_{0}+\mathbf{i} f_{1}+\mathbf{j} f_{2}+\mathbf{k} f_{3}, \quad g=g_{0}+\mathbf{i} g_{1}+\mathbf{j} g_{2}+\mathbf{k} g_{3} .
$$

If $\mathscr{F}_{q}\{g\} \in L^{2}\left(\mathbb{R}^{2} ; \mathbb{R}\right)$, then (40) takes the form

$$
\begin{aligned}
\mathscr{F}_{q}\{f \star g\}(\boldsymbol{\omega})= & \left(\mathscr{F}_{q}\left\{f_{0}\right\}(\boldsymbol{\omega})+\mathbf{i} \mathscr{F}_{q}\left\{f_{1}\right\}(\boldsymbol{\omega})\right) \mathscr{F}_{q}\{g\}(\boldsymbol{\omega}) \\
& +\left(\mathbf{j} \mathscr{F}_{q}\left\{f_{2}\right\}\left(-\omega_{1}, \omega_{2}\right)\right. \\
& \left.+\mathbf{k} \mathscr{F}_{q}\left\{f_{3}\right\}\left(-\omega_{1}, \omega_{2}\right)\right) \mathscr{F}_{q}\{g\}\left(-\omega_{1}, \omega_{2}\right) .
\end{aligned}
$$

On the other hand, if $\mathscr{F}_{q}\{f\} \in L^{2}\left(\mathbb{R}^{2} ; \mathbb{R}\right)$, then

$$
\begin{aligned}
\mathscr{F}_{q}\{f \star g\}(\boldsymbol{\omega}) \\
=\mathscr{F}_{q}\{f\}(\boldsymbol{\omega})\left(\mathscr{F}_{q}\left\{g_{0}\right\}(\boldsymbol{\omega})+\mathbf{j} \mathscr{F}_{q}\left\{g_{2}\right\}\left(-\omega_{1}, \omega_{2}\right)\right) \\
\quad+\mathscr{F}_{q}\{f\}\left(\omega_{1},-\omega_{2}\right)\left(\mathbf{i} \mathscr{F}_{q}\left\{g_{1}\right\}(\boldsymbol{\omega})+\mathbf{k} \mathscr{F}_{q}\left\{g_{3}\right\}\left(-\omega_{1}, \omega_{2}\right)\right) .
\end{aligned}
$$

Proof. We only prove expression (44) of Lemma 14, with the other being similar. Following the steps of (41) we immediately get

$$
\begin{aligned}
\mathscr{F}_{q}\{f \star g\}(\boldsymbol{\omega}) \\
=\iint_{\mathbb{R}^{2}} e^{-\mathbf{i} \omega_{1}\left(y_{1}+z_{1}\right)} f(\mathbf{y}) g(\mathbf{z}) e^{-\mathbf{j} \omega_{2}\left(y_{2}+z_{2}\right)} d \mathbf{z} d \mathbf{y} \\
=\iint_{\mathbb{R}^{2}} e^{-\mathbf{i} \omega_{1}\left(y_{1}+z_{1}\right)} \\
\quad \times\left(\left\{f_{0}(\mathbf{y})+\mathbf{i} f_{1}(\mathbf{y})\right\}+\mathbf{j} f_{2}(\mathbf{y})+\mathbf{k} f_{3}(\mathbf{y})\right)
\end{aligned}
$$




$$
\begin{aligned}
& \quad \times g(\mathbf{z}) e^{-\mathbf{j} \omega_{2}\left(y_{2}+z_{2}\right)} d \mathbf{z} d \mathbf{y} \\
& =\iint_{\mathbb{R}^{2}} e^{-\mathbf{i} \omega_{1}\left(y_{1}+z_{1}\right)}\left(f_{0}(\mathbf{y})+\mathbf{i} f_{1}(\mathbf{y})\right) \\
& \quad \times g(\mathbf{z}) e^{-\mathbf{j} \omega_{2}\left(y_{2}+z_{2}\right)} d \mathbf{z} d \mathbf{y} \\
& +\iint_{\mathbb{R}^{2}} e^{-\mathbf{i} \omega_{1}\left(y_{1}+z_{1}\right)}\left(\mathbf{j} f_{2}(\mathbf{y})+\mathbf{k} f_{3}(\mathbf{y})\right) \\
& \quad \times g(\mathbf{z}) e^{-\mathbf{j} \omega_{2}\left(y_{2}+z_{2}\right)} d \mathbf{z} d \mathbf{y} \\
& =\int_{\mathbb{R}^{2}} e^{-\mathbf{i} \omega_{1} y_{1}}\left(f_{0}(\mathbf{y})+\mathbf{i} f_{1}(\mathbf{y})\right) \mathscr{F}_{q}\{g\}(\boldsymbol{\omega}) e^{-\mathbf{j} \omega_{2} y_{2}} d \mathbf{y} \\
& +\int_{\mathbb{R}^{2}} e^{-\mathbf{i} \omega_{1} y_{1}}\left(\mathbf{j} f_{2}(\mathbf{y})+\mathbf{k} f_{3}(\mathbf{y})\right) \\
& \quad \times \mathscr{F}_{q}\{g\}\left(-\omega_{1}, \omega_{2}\right) e^{-\mathbf{j} \omega_{2} y_{2}} d \mathbf{y} \\
& =\left(\mathscr{F}_{q}\left\{f_{0}\right\}(\boldsymbol{\omega})+\mathbf{i} \mathscr{F}_{q}\left\{f_{1}\right\}(\boldsymbol{\omega})\right) \mathscr{F}_{q}\{g\}(\boldsymbol{\omega}) \\
& +\left(\mathbf{j} \mathscr{F}_{q}\left\{f_{2}\right\}\left(-\omega_{1}, \omega_{2}\right)+\mathbf{k} \mathscr{F}_{q}\left\{f_{3}\right\}\left(-\omega_{1}, \omega_{2}\right)\right) \\
& \quad \times \mathscr{F}_{q}\{g\}\left(-\omega_{1}, \omega_{2}\right),
\end{aligned}
$$

which was to be proven.

Lemma 15. Let $f, g \in L^{2}\left(\mathbb{R}^{2} ; \mathbb{H}\right)$, where

$$
f=f_{0}+\mathbf{i} f_{1}, \quad g=g_{0}+\mathbf{i} g_{1}+\mathbf{j} g_{2}+\mathbf{k} g_{3} .
$$

If $\mathscr{F}_{q}\{f\}, \mathscr{F}_{q}\{g\} \in L^{2}\left(\mathbb{R}^{2} ; \mathbb{R}\right)$, then

$$
\begin{aligned}
\mathscr{F}_{q}\{f \star g\}(\omega) & =\mathscr{F}_{q}\{f\}(\boldsymbol{\omega}) \mathscr{F}_{q}\{g\}(\boldsymbol{\omega}) \\
& =\mathscr{F}_{q}\{g\}(\boldsymbol{\omega}) \mathscr{F}_{q}\{f\}(\boldsymbol{\omega}),
\end{aligned}
$$

which is of the same form as a convolution of the classical Fourier transform [20].

Remark 16. It is important to notice that, if $f, g \in L^{2}\left(\mathbb{R}^{2} ; \mathbb{H}\right)$, where

$$
f=\mathbf{j} f_{2}+\mathbf{k} f_{3}, \quad g=g_{0}+\mathbf{i} g_{1}+\mathbf{j} g_{2}+\mathbf{k} g_{3},
$$

then Lemma 15 reduces to

$$
\begin{aligned}
\mathscr{F}_{q}\{f \star g\}(\boldsymbol{\omega}) & =\mathscr{F}_{q}\{f\}(\boldsymbol{\omega}) \mathscr{F}_{q}\{g\}\left(-\omega_{1}, \omega_{2}\right) \\
& =\mathscr{F}_{q}\{g\}\left(-\omega_{1}, \omega_{2}\right) \mathscr{F}_{q}\{f\}(\boldsymbol{\omega}),
\end{aligned}
$$

where $\mathscr{F}_{q}\{f\}, \mathscr{F}_{q}\{g\} \in L^{2}\left(\mathbb{R}^{2} ; \mathbb{R}\right)$.

Table 2 compares convolution theorems of the QFT and classical FT for $f, g \in L^{2}\left(\mathbb{R}^{2} ; \mathbb{H}\right)$.
TABLE 2: Comparison of convolution theorems of the QFT and classical FT for $f, g \in L^{2}\left(\mathbb{R}^{2} ; \mathbb{U}\right)$.

\begin{tabular}{ll}
\hline Assumptions on quaternion functions & QFT of convolution \\
\hline $\mathscr{F}_{q}\{f\}, \mathscr{F}_{q}\{g\} \in L^{2}\left(\mathbb{R}^{2} ; \mathbb{H}\right)$ & QFT $\neq$ classical FT \\
$\mathscr{F}_{q}\{f\} \in L^{2}\left(\mathbb{R}^{2} ; \mathbb{R}\right)$ and $\mathscr{F}_{q}\{g\} \in L^{2}\left(\mathbb{R}^{2} ; \mathbb{H}\right)$ & QFT $\neq$ classical FT \\
$\mathscr{F}_{q}\{f\} \in L^{2}\left(\mathbb{R}^{2} ; \mathbb{H}\right)$ and $\mathscr{F}_{{ }_{q}}\{g\} \in L^{2}\left(\mathbb{R}^{2} ; \mathbb{R}\right)$ & QFT $\neq$ classical FT \\
$f=f_{0}+\mathbf{i} f_{1}$ and $\mathscr{F}_{{ }_{q}}\{f\}, \mathscr{F}_{q}\{g\} \in L^{2}\left(\mathbb{R}^{2} ; \mathbb{R}\right)$ & QFT $=$ classical FT \\
$f=\mathbf{j} f_{2}+\mathbf{k} f_{3}$ and $\mathscr{F}_{q}\{f\}, \mathscr{F}_{q}\{g\} \in L^{2}\left(\mathbb{R}^{2} ; \mathbb{R}\right)$ & QFT $=$ classical FT \\
\hline
\end{tabular}

The following theorem is useful for solving the heat equation in quaternion algebra.

Theorem 17. If $f, g \in L^{2}\left(\mathbb{R}^{2} ; \mathbb{H}\right)$ and $\mathscr{F}_{q}\{g\} \in L^{2}\left(\mathbb{R}^{2} ; \mathbb{R}\right)$, then

$$
\begin{aligned}
\mathscr{F}_{q}^{-1}\left[\mathscr{F}_{q}\{f\} \mathscr{F}_{q}\{g\}\right](\mathbf{x}) \\
=\left(f_{0} \star g\right)(\mathbf{x})+\left(\mathbf{i} f_{1} \star g\right)(\mathbf{x}) \\
\quad+\left(\mathbf{j} f_{2} \star g\right)\left(-x_{1}, x_{2}\right)+\left(\mathbf{k} f_{3} \star g\right)\left(-x_{1}, x_{2}\right) .
\end{aligned}
$$

Proof. By the QFT inversion, we get, after some simplification,

$$
\mathscr{F}_{q}^{-1}\left[\mathscr{F}_{q}\{f\} \mathscr{F}_{q}\{g\}\right](\mathbf{x})
$$

$\stackrel{(23)}{=} \frac{1}{(2 \pi)^{2}} \iint_{\mathbb{R}^{2}} e^{\mathbf{i} \omega_{1} x_{1}} e^{-\mathbf{i} \omega_{1} y_{1}} f(\mathbf{y}) e^{-\mathbf{j} \omega_{2} y_{2}} d \mathbf{y}$

$$
\begin{aligned}
& \times \mathscr{F}_{q}\{g\}(\boldsymbol{\omega}) e^{\mathrm{j} \omega_{2} x_{2}} d \boldsymbol{\omega} \\
& =\frac{1}{(2 \pi)^{2}} \iint_{\mathbb{R}^{2}} e^{\mathbf{i} \omega_{1}\left(x_{1}-y_{1}\right)} f(\mathbf{y}) \\
& \times \mathscr{F}_{q}\{g\}(\boldsymbol{\omega}) e^{\mathbf{j} \omega_{2}\left(x_{2}-y_{2}\right)} d \mathbf{y} d \boldsymbol{\omega} \\
& =\frac{1}{(2 \pi)^{2}} \iint_{\mathbb{R}^{2}} e^{\mathbf{i} \omega_{1}\left(x_{1}-y_{1}\right)} \\
& \times\left(f_{0}(\mathbf{y})+\mathbf{i} f_{1}(\mathbf{y})+\mathbf{j} f_{2}(\mathbf{y})+\mathbf{k} f_{3}(\mathbf{y})\right) \\
& \times \mathscr{F}_{q}\{g\}(\boldsymbol{\omega}) e^{\mathbf{j} \omega_{2}\left(x_{2}-y_{2}\right)} d \mathbf{y} d \boldsymbol{\omega} \\
& =\frac{1}{(2 \pi)^{2}} \iint_{\mathbb{R}^{2}} e^{\mathbf{i} \omega_{1}\left(x_{1}-y_{1}\right)}\left(f_{0}(\mathbf{y})+\mathbf{i} f_{1}(\mathbf{y})\right) \\
& +e^{\mathbf{i} \omega_{1}\left(x_{1}-y_{1}\right)}\left(\mathbf{j} f_{2}(\mathbf{y})+\mathbf{k} f_{3}(\mathbf{y})\right) \\
& \times \mathscr{F}_{q}\{g\}(\boldsymbol{\omega}) e^{\mathbf{j} \omega_{2}\left(x_{2}-y_{2}\right)} d \mathbf{y} d \boldsymbol{\omega} \\
& =\int_{\mathbb{R}^{2}}\left(f_{0}(\mathbf{y})+\mathbf{i} f_{1}(\mathbf{y})\right) g(\mathbf{x}-\mathbf{y}) d \mathbf{y} \\
& +\int_{\mathbb{R}^{2}}\left(\mathbf{j} f_{2}(\mathbf{y})+\mathbf{k} f_{3}(\mathbf{y})\right) g\left(-\left(x_{1}-y_{1}\right), x_{2}-y_{2}\right) d \mathbf{y} \\
& =\left(f_{0} \star g\right)(\mathbf{x})+\left(\mathbf{i} f_{1} \star g\right)(\mathbf{x})+\left(\mathbf{j} f_{2} \star g\right)\left(-x_{1}, x_{2}\right) \\
& +\left(\mathbf{k} f_{3} \star g\right)\left(-x_{1}, x_{2}\right) \text {, }
\end{aligned}
$$


where, in the second line, we have used the assumption $\mathscr{F}_{q}\{g\} \in L^{2}\left(\mathbb{R}^{2} ; \mathbb{R}\right)$. This completes the proof of (51).

As an immediate consequence of Theorem 17, we get the following corollaries.

Corollary 18. If $f, g \in L^{2}\left(\mathbb{R}^{2} ; \mathbb{H}\right)$ and $\mathscr{F}_{q}\{g\} \in L^{2}\left(\mathbb{R}^{2} ; \mathbb{R}\right)$, where

$$
f=f_{0}+\mathbf{i} f_{1}, \quad g=g_{0}+\mathbf{i} g_{1}+\mathbf{j} g_{2}+\mathbf{k} g_{3},
$$

then (51) reduces to

$$
\mathscr{F}_{q}^{-1}\left[\mathscr{F}_{q}\{f\} \mathscr{F}_{q}\{g\}\right](\mathbf{x})=(f \star g)(\mathbf{x}) .
$$

Corollary 19. Let

$$
f(\mathbf{x})= \begin{cases}e^{-\left(x_{1}+x_{2}\right)}, & \text { if } x_{1}>0, x_{2}>0 \\ 0, & \text { otherwise }\end{cases}
$$

And consider the quaternionic Gabor filter

$$
g(\mathbf{x})=e^{\mathbf{i} u_{0} x_{1}} e^{\mathbf{j} v_{0} x_{2}} e^{-(1 / 2)|\mathbf{x}|^{2}} .
$$

Then,

$$
\begin{aligned}
\mathscr{F}_{q}\{f \star g\}(\boldsymbol{\omega}) \\
=\frac{e^{-(1 / 2)\left(\left(\omega_{1}-u_{0}\right)^{2}+\left(\omega_{2}-v_{0}\right)^{2}\right)}\left(1-\mathbf{i} \omega_{1}-\mathbf{j} \omega_{2}-\mathbf{k} \omega_{1} \omega_{2}\right)}{(2 \pi)^{2}\left(1+\omega_{1}^{2}+\omega_{2}^{2}+\omega_{1}^{2} \omega_{2}^{2}\right)} .
\end{aligned}
$$

Proof. The QFT of $f$ is given by

$$
\mathscr{F}_{q}\{f\}(\boldsymbol{\omega})=\frac{1-\mathbf{i} \omega_{1}-\mathbf{j} \omega_{2}-\mathbf{k} \omega_{1} \omega_{2}}{(2 \pi)^{2}\left(1+\omega_{1}^{2}+\omega_{2}^{2}+\omega_{1}^{2} \omega_{2}^{2}\right)},
$$

and the QFT of $g$ is given by

$$
\mathscr{F}_{q}\{g\}(\boldsymbol{\omega})=e^{-(1 / 2)\left(\left(\omega_{1}-u_{0}\right)^{2}+\left(\omega_{2}-v_{0}\right)^{2}\right)} .
$$

Therefore, using Corollary 18, we obtain (57).

\section{Applications of QFT}

In [19], the authors proposed to use quaternions in order to define a Fourier transform applicable to color images. Their framework makes it possible to compute a single, holistic, Fourier transform which treats a color image as a vector field. In image processing, taking a given image as the initial value, the forward solution to the heat equation or a diffusion equation in general, produces blurred images and the backward solution produces sharpen images for example, see [21, pages 342-350].

In this section, we present two applications of QFT to partial differential equations in quaternion algebra.
4.1. Hypoellipticity. In this paper, since we only deal with QFT in the $L^{2}\left(\mathbb{R}^{2} ; \mathbb{U}\right)$ framework, we will discuss the hypoellipticity in this framework; that is, we will only deal with $L^{2}\left(\mathbb{R}^{2} ; \mathbb{H}\right)$ solutions for linear partial differential operators with constant quaternion coefficients:

$$
P(\partial)=\sum_{0 \leq|\boldsymbol{\alpha}| \leq n} a_{\boldsymbol{\alpha}} \partial^{\boldsymbol{\alpha}} b_{\boldsymbol{\alpha}}, \quad a_{\boldsymbol{\alpha}}, b_{\boldsymbol{\alpha}} \in \mathbb{H} .
$$

The noncommutativity of quaternion gives different aspects of $P(\partial)$ with constant complex coefficients $a_{\boldsymbol{\alpha}}, b_{\boldsymbol{\alpha}} \in \mathbb{C}$.

Example 20. Let $P(\partial)=\partial_{x_{1}} b, b \in \mathbb{H}$, and $f(\mathbf{x}), g(\mathbf{x}) \in$ $C^{1}\left(\mathbb{R}^{2} ; \mathbb{H}\right)$.

(i) Since $\partial_{x_{1}}(f(\mathbf{x}) g(\mathbf{x}))=\left(\partial_{x_{1}} f(\mathbf{x})\right) g(\mathbf{x})+f(\mathbf{x})\left(\partial_{x_{1}} g(\mathbf{x})\right)$, we have $P(\partial)(f(\mathbf{x}) g(\mathbf{x}))=(P(\partial) f(\mathbf{x})) g(\mathbf{x})+f(\mathbf{x})$ $(P(\partial) g(\mathbf{x}))$ when $b \in \mathbb{C}$. But, when $b \in \mathbb{U} \backslash \mathbb{C}$, as $b f(\mathbf{x}) \neq f(\mathbf{x}) b$ in general, we cannot have $P(\partial)$ $(f(\mathbf{x}) g(\mathbf{x}))=(P(\partial) f(\mathbf{x})) g(\mathbf{x})+f(\mathbf{x})(P(\partial) g(\mathbf{x}))$ in general.

(ii) We have $P(\partial) f \star g=f \star P(\partial) g$ when $b \in \mathbb{C}$. But, by the same reason as (i), when $b \in \mathbb{H} \backslash \mathbb{C}$, we cannot have $P(\partial) f \star g=f \star P(\partial) g$ in general.

Let us start with the definition of our $L^{2}\left(\mathbb{R}^{2} ; \mathbb{H}\right)$ version of hypoellipticity (compared to [22, page 110]).

Definition 21. The linear partial differential operator $P(\partial)$ in $\mathbb{R}^{2}$ is said to be $L^{2}\left(\mathbb{R}^{2} ; \mathbb{H}\right)$-hypoelliptic if, given any subset $U$ of $\mathbb{R}^{2}$ and any solution $u$ in $L^{2}\left(\mathbb{R}^{2} ; \mathbb{U}\right)$ such that $P(\partial) u$ is a $C^{\infty}$ function in $U$, then all its components $u\left(u_{i}, i=0,1,2,3\right)$ are a $C^{\infty}$ function in $U$.

Definition 22. Given a linear partial differential operator $P(\partial)$ of (60) with the quaternion constant coefficients. One says that a solution $E(\mathbf{x})$ of $P(\partial) u=\delta$, where $\delta$ is the delta function, is called a fundamental solution of $P(\partial)$.

Let $A$ and $B$ be subsets of $\mathbb{R}^{2}$. Define the sum $A+B$ by $A+B=\left\{\mathbf{x}+\mathbf{y} \in \mathbb{R}^{2} ; \mathbf{x} \in A, \mathbf{y} \in B\right\}$.

Theorem 23. Assume that there is one fundamental solution $E(\mathbf{x})$ of $P(\partial)$ which is a $C^{\infty}$ function in $\mathbb{R}^{2} \backslash\{0\}$, and the identities

$$
P(\partial)(f \star g)=P(\partial) f \star g=f \star P(\partial) g
$$

are satisfied for arbitrary sufficiently smooth quaternionvalued functions $f$ and $g$ such that $g f$ is a compactly supported $C^{\infty}$ quaternion function with $a_{\alpha}$ of $P(\partial)$ being quaternion constant coefficients and $b_{\alpha}$ of $P(\partial)$ being real constant coefficients. Then, the linear partial differential operator $P(\partial)$ is $L^{2}\left(\mathbb{R}^{2} ; \mathbb{H}\right)$ hypoelliptic in $\mathbb{R}^{2}$.

Proof. Firstly, let $U$ be an arbitrary open subset of $\mathbb{R}^{2}$ and $u$ a solution in $U$ with values in $\mathbb{H}$ such that $f=P(\partial) u$ is a $C^{\infty}$ function in $U$. Let $\mathbf{x}_{0}$ be an arbitrary point in $U$. It will suffice to show that $u$ is a $C^{\infty}$ function in some open neighborhood 
of $\mathbf{x}_{0}$. Take an open disc $D_{\eta}\left(\mathbf{x}_{0}\right)=\left\{\mathbf{x} \in \mathbb{R}^{2} ;\left|\mathbf{x}-\mathbf{x}_{0}\right|<\eta\right\}$ such that $\overline{D_{\eta}\left(\mathbf{x}_{0}\right)} \subset U$. There exists a function $g \in C^{\infty}(U ; \mathbb{R})$ such that supp $g \subset U$ and $g=1$ in $D_{\eta}\left(\mathbf{x}_{0}\right)$. Then, we have

$$
P(\partial)(g u)=g P(\partial) u+v=g f+v,
$$

where every term of $v$ contains a derivative of $g$ of nonzero order; therefore $v=0$, where the derivatives of $g$ vanish, especially in $D_{\eta}\left(\mathbf{x}_{0}\right)$ and outside of supp $g$. For the fundamental solution $E(\mathbf{x})$, the hypothesis (61) implies

$$
E \star P(\partial)(g u)=\{P(\partial) E\} \star(g u)=g u .
$$

Hence

$$
g u=E \star(g f)+E \star v .
$$

But $g f$ is a compactly supported $C^{\infty}$ function and the convolution of any function with any compactly supported $C^{\infty}$ function is a $C^{\infty}$ function. Therefore, it suffices to show that $E \star v$ is a $C^{\infty}$ function in an open neighborhood of $\mathbf{x}_{0}$, because $g u$ is also a $C^{\infty}$ function in an open neighborhood of $\mathbf{x}_{0}$ and $g u=u$ in $D_{\eta}\left(\mathbf{x}_{0}\right)$.

Finally, we will show that $E \star v$ is a $C^{\infty}$ function in an open neighborhood of $\mathbf{x}_{0}$. Let us select $\varepsilon>0$ such that $\varepsilon<$ $1 / 2 \eta$. Then, the open disc $D_{\varepsilon}\left(\mathbf{x}_{0}\right)$ is a neighborhood of $\mathbf{x}_{0}$. Let $\zeta_{\varepsilon}(\mathbf{x}) \in C^{\infty}\left(\mathbb{R}^{2} ; \mathbb{R}\right)$, another cutoff function, be equal to one for $|\mathbf{x}|<\varepsilon / 2$ and to zero for $\left|\mathbf{x}-\mathbf{x}_{0}\right|>\varepsilon$. We have

$$
E \star v=\left(\zeta_{\varepsilon} E\right) \star v+\left\{\left(1-\zeta_{\varepsilon}\right) E\right\} \star v .
$$

The hypothesis implies that $\left(1-\zeta_{\varepsilon}\right) E \in C^{\infty}\left(\mathbb{R}^{2} ; \mathbb{U}\right)$, and therefore $\left(1-\zeta_{\varepsilon}\right) E \star v \in C^{\infty}\left(\mathbb{R}^{2} ; \mathbb{A}\right)$. Since

$$
\operatorname{supp}\left\{\left(\zeta_{\varepsilon} E\right) \star v\right\} \subset \operatorname{supp}\left(\zeta_{\varepsilon} E\right)+\operatorname{supp} v,
$$

$\operatorname{supp}\left\{\left(\zeta_{\varepsilon} E\right) \star v\right\}$ is contained in the $\varepsilon$-neighborhood of supp $v$. We have already seen that $v=0 \in D_{\eta}\left(\mathbf{x}_{0}\right)$. Hence, $\left(\zeta_{\varepsilon} E\right) \star v$ vanishes in $D_{\varepsilon}\left(\mathbf{x}_{0}\right)$, and, therefore, $E \star v$ is a $C^{\infty}$ function in $D_{\varepsilon}\left(\mathbf{x}_{0}\right)$.

4.2. Parabolic Initial Value Problem. Let us consider the parabolic initial value problem

$$
\frac{\partial}{\partial t} u-\nabla^{2} u=0, \quad \text { on } \mathbb{R}^{2} \times(0, \infty)
$$

with

$$
u(\mathbf{x}, 0)=f(\mathbf{x}), \quad f \in \mathcal{S}\left(\mathbb{R}^{2} ; \mathbb{\sharp}\right),
$$

where $\mathcal{S}\left(\mathbb{R}^{2} ; \mathbb{H}\right)$ is the quaternion Schwartz space. Applying the QFT, we easily obtain

$$
\begin{aligned}
\mathscr{F}_{q}\left\{u_{t}\right\} & =\left(\mathbf{i} \omega_{1}\right)^{2} \mathscr{F}_{q}\{u\}(\boldsymbol{\omega})+\mathscr{F}_{q}\{u\}(\boldsymbol{\omega})\left(\mathbf{j} \omega_{2}\right)^{2} \\
& =-|\boldsymbol{\omega}|^{2} \mathscr{F}_{q}\{u\}(\boldsymbol{\omega}) .
\end{aligned}
$$

The general solution of (69) is given by

$$
\mathscr{F}_{q}\{u\}(\boldsymbol{\omega}, t)=C e^{-|\boldsymbol{\omega}|^{2} t},
$$

where $C$ is a quaternion constant. We impose the initial condition $\mathscr{F}_{q}\{u\}(\boldsymbol{\omega}, 0)=\mathscr{F}_{q}\{f\}(\boldsymbol{\omega})$ to obtain

$$
\mathscr{F}_{q}\{u\}(\boldsymbol{\omega}, t)=e^{-|\boldsymbol{\omega}|^{2} t} \mathscr{F}_{q}\{f\}(\boldsymbol{\omega}) .
$$

Notice that the QFT of a Gaussian quaternion function is also a Gaussian quaternion function (compared to Bahri et al. [13]). Hence

$$
\frac{1}{4 \pi t} \mathscr{F}_{q}\left\{\left(e^{-|\mathbf{x}|^{2} /(4 t)}\right)\right\}=e^{-|\boldsymbol{\omega}|^{2} t} .
$$

Applying the inverse QFT, we have

$$
\begin{aligned}
u(\mathbf{x}, t) & =\mathscr{F}_{q}^{-1}\left[e^{-|\omega|^{2} t} \mathscr{F}_{q}\{f\}\right](\mathbf{x}) \\
& =\frac{1}{4 \pi t} \mathscr{F}_{q}^{-1}\left[\mathscr{F}_{q}\{f\} \mathscr{F}_{q}\left\{e^{-|\mathbf{x}|^{2} /(4 t)}\right\}\right](\mathbf{x}) .
\end{aligned}
$$

Since

$$
\mathscr{F}_{q}\left\{e^{-|\mathbf{x}|^{2} /(4 t)}\right\}(\boldsymbol{\omega})=4 \pi t e^{-|\boldsymbol{\omega}|^{2} t} \in L^{2}\left(\mathbb{R}^{2} ; \mathbb{R}\right),
$$

then we can apply the convolution theorem of (51) to get

$$
\begin{aligned}
u(\mathbf{x}, t)= & \left(f_{0} \star K_{t}\right)(\mathbf{x})+\left(\mathbf{i} f_{1} \star K_{t}\right)(\mathbf{x}) \\
& +\left(\mathbf{j} f_{2} \star K_{t}\right)\left(-x_{1}, x_{2}\right)+\left(\mathbf{k} f_{3} \star K_{t}\right)\left(-x_{1}, x_{2}\right),
\end{aligned}
$$

where $K_{t}=(1 / 4 \pi t) e^{-|\mathbf{x}|^{2} /(4 t)}$, and $f_{i} \in L^{2}\left(\mathbb{R}^{2} ; \mathbb{R}\right), i=0,1,2,3$. By Definition 6 of the convolution, we finally obtain

$$
\begin{aligned}
u(\mathbf{x}, t)= & \frac{1}{4 \pi t} \int_{\mathbb{R}^{2}} f_{0}(\mathbf{y}) e^{-\left(\left(x_{1}-y_{1}\right)^{2}+\left(x_{2}-y_{2}\right)^{2}\right) /(4 t)} d \mathbf{y} \\
& +\frac{\mathbf{i}}{4 \pi t} \int_{\mathbb{R}^{2}} f_{1}(\mathbf{y}) e^{-\left(\left(x_{1}-y_{1}\right)^{2}+\left(x_{2}-y_{2}\right)^{2}\right) /(4 t)} d \mathbf{y} \\
& +\frac{\mathbf{j}}{4 \pi t} \int_{\mathbb{R}^{2}} f_{2}(\mathbf{y}) e^{-\left(\left(-x_{1}-y_{1}\right)^{2}+\left(x_{2}-y_{2}\right)^{2}\right) /(4 t)} d \mathbf{y} \\
& +\frac{\mathbf{k}}{4 \pi t} \int_{\mathbb{R}^{2}} f_{3}(\mathbf{y}) e^{-\left(\left(-x_{1}-y_{1}\right)^{2}+\left(x_{2}-y_{2}\right)^{2}\right) /(4 t)} d \mathbf{y} .
\end{aligned}
$$

In an actual application, one often takes the quaternionic Gabor filter (see $[6,10])$ as

$$
f(\mathbf{x})=e^{\mathbf{i} u_{0} x_{1}} e^{-\left(x_{1}^{2}+x_{2}^{2}\right)} e^{\mathbf{j} v_{0} x_{2}} .
$$

Therefore, the above identity will reduce to

$$
\begin{array}{r}
u(\mathbf{x}, t)=\frac{1}{4 \pi t} \int_{\mathbb{R}^{2}} \cos \left(u_{0} x_{1}\right) \cos \left(v_{0} x_{2}\right) e^{-\left(x_{1}^{2}+x_{2}^{2}\right)} \\
\times e^{-\left(\left(x_{1}-y_{1}\right)^{2}+\left(x_{2}-y_{2}\right)^{2}\right) /(4 t)} d \mathbf{y},
\end{array}
$$




$$
\begin{gathered}
+\frac{\mathbf{i}}{4 \pi t} \int_{\mathbb{R}^{2}} \sin \left(u_{0} x_{1}\right) \cos \left(v_{0} x_{2}\right) e^{-\left(x_{1}^{2}+x_{2}^{2}\right)} \\
\times e^{-\left(\left(x_{1}-y_{1}\right)^{2}+\left(x_{2}-y_{2}\right)^{2}\right) /(4 t)} d \mathbf{y}, \\
+\frac{\mathbf{j}}{4 \pi t} \int_{\mathbb{R}^{2}} \cos \left(u_{0} x_{1}\right) \sin \left(v_{0} x_{2}\right) e^{-\left(x_{1}^{2}+x_{2}^{2}\right)} \\
\times e^{-\left(\left(-x_{1}-y_{1}\right)^{2}+\left(x_{2}-y_{2}\right)^{2}\right) /(4 t)} d \mathbf{y}, \\
+\frac{\mathbf{k}}{4 \pi t} \int_{\mathbb{R}^{2}} \sin \left(u_{0} x_{1}\right) \sin \left(v_{0} x_{2}\right) e^{-\left(x_{1}^{2}+x_{2}^{2}\right)} \\
\times e^{-\left(\left(-x_{1}-y_{1}\right)^{2}+\left(x_{2}-y_{2}\right)^{2}\right) /(4 t)} d \mathbf{y} .
\end{gathered}
$$

\section{Conclusion}

Due to the non-commutative property of quaternion multiplication, there are three different types of two-dimensional QFTs. These three QFTs are the so-called left-sided QFT, right-sided QFT, and a double-sided QFT, respectively. In this work, we have established convolution theorem of the double-sided QFT applied to real fields $f: \mathbb{R}^{2} \rightarrow \mathbb{R}$ and quaternion fields $f: \mathbb{R}^{2} \rightarrow \mathbb{H}$. Some important properties of the QFT convolution are investigated. We have shown that the QFT convolution is useful to study hypoellipticity and to solve the heat equation in quaternion algebra framework. It can easily be seen that the solution of generalized heat equation is extension of solution of the classical heat equation.

The future work will establish the convolution theorems of the right-sided QFT. We compare some properties of the convolution theorems of the two types of QFTs. We will apply the properties to find the solution of partial differential equations in quaternion algebra framework. The solutions of generalized partial differential equations using the properties of the three types of two-dimensional QFTs will be compared too.

\section{Acknowledgments}

The authors would like to thank the reviewers whose deep and extensive comments greatly contributed to improve this paper. The first author is partially supported by Hibah Penelitian Kompetisi Internal Tahun 2013 (no. 110/UN4.42/LK.26/SP-UH/2013) from the Hasanuddin University, Indonesia. The second author is partially supported by JSPS.KAKENHI (C)22540130 and (C)25400202 of Japan and the third author is partially supported by NSERC of Canada.

\section{References}

[1] J. Ebling and G. Scheuermann, "Clifford Fourier transform on vector fields," IEEE Transactionson Visualization and Computer Graphics, vol. 11, no. 4, pp. 469-479, 2005.

[2] E. Hitzer and B. Mawardi, "Clifford Fourier transform on multivector fields and uncertainty principles for dimensions $n=2(\bmod 4)$ and $n=3(\bmod 4), "$ Advances in Applied Clifford Algebras, vol. 18, no. 3-4, pp. 715-736, 2008.

[3] B. Mawardi and E. Hitzer, "Clifford Fourier transformation and uncertainty principle for the Clifford geometric algebra $\mathrm{Cl}_{3,0}$," Advances in Applied Clifford Algebras, vol. 16, no. 1, pp. 41-61, 2006.

[4] H. de Bie and N. de Schepper, "Fractional Fourier transform of hypercomplex signals," Signal, Image and Video Processing, vol. 6, no. 3, pp. 381-388, 2012.

[5] X. Guanlei, W. Xiaotong, and X. Xiagang, "Fractional quaternion Fourier transform, convolutionand correlation," Signal Processing, vol. 88, no. 10, pp. 2511-2517, 2008.

[6] M. Bahri, E. Hitzer, R. Ashino, and R. Vaillancourt, "Windowed Fourier transform of two-dimensional quaternionic signals," Applied Mathematics and Computation, vol. 216, no. 8, pp. 23662379, 2010.

[7] M. Bahri, R. Ashino, and R. Vaillancourt, "Two-dimensional quaternion wavelet transform," Applied Mathematics and Computation, vol. 218, no. 1, pp. 10-21, 2011.

[8] M. Bahri, "Quaternion algebra-valued wavelet transform," Applied Mathematical Sciences, vol. 5, no. 71, pp. 3531-3540, 2011.

[9] S. J. Sangwine, "Biquaternion (complexified quaternion) roots of -1 ," Advances in Applied Clifford Algebras, vol. 16, no. 1, pp. 63-68, 2006.

[10] T. Bülow, Hypercomplex spectral signal representations for the processing and analysis of images [Ph.D. thesis], University of Kiel, Kiel, Germany, 1999.

[11] T. A. Ell, "Quaternion-Fourier transformations for analysis of two-dimensional lineartime-invariant partial differential systems," in Proceedings of the 32nd IEEE Conference on Decision and Control, vol. 2, pp. 1830-1841, San Antonio, Tex, USA, December 1993.

[12] E. Hitzer, "Quaternion Fourier transform on quaternion fields and generalizations," Advances in Applied Clifford Algebras, vol. 17, no. 3, pp. 497-517, 2007.

[13] M. Bahri, E. Hitzer, A. Hayashi, and R. Ashino, "An uncertainty principle for quaternion Fourier transform," Computers \& Mathematics with Applications, vol. 56, no. 9, pp. 2411-2417, 2008.

[14] D. Assefa, L. Mansinha, K. F. Tiampo, H. Rasmussen, and K. Abdella, "Local quaternion Fourier transform and color images texture analysis," Signal Processing, vol. 90, no. 6, pp. 1825-1835, 2010.

[15] R. Bujack, G. Scheuermann, and E. Hitzer, "A general geometric fourier transform convolution theorem," Advances in Applied Clifford Algebras, vol. 23, no. 1, pp. 15-38, 2013.

[16] R. Bujack, G. Scheuermann, and E. Hitzer, "A general geometric Fourier transform," in Proceedings of the 9th International Conference on Clifford Algebras and Their Applications in Mathematical Physics (ICCA '11), K. Guerlebeck, Ed., Weimar, Germany, July 2011.

[17] E. Hitzer, "Directional uncertainty principle for quaternion Fourier transform," Advances in Applied Clifford Algebras, vol. 20, no. 2, pp. 271-284, 2010.

[18] G. B. Folland, Real Analysis: Modern Techniques and Their Applications, Pure and Applied Mathematics, John Wiley \& Sons, New York, NY, USA, 2nd edition, 1999.

[19] T. A. Ell and S. J. Sangwine, "Hypercomplex Fourier transforms of color images," IEEE Transactions on Image Processing, vol. 16, no. 1, pp. 22-35, 2007. 
[20] S. Mallat, A Wavelet Tour of Signal Processing, Academic Press, San Diego, Calif, USA, 2nd edition, 1999.

[21] M. Petrou and C. Petrou, Image Processing: The Fundamentals, John Wiley \& Sons, West Sussex, UK, 2nd edition, 2010.

[22] L. Hörmander, The Analysis of Linear Partial Differential Operators I: Distribution Theory and Fourier Analysis, Springer, Berlin, Germany, 1983. 


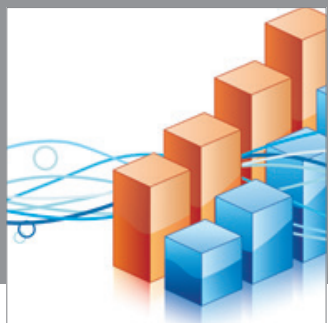

Advances in

Operations Research

mansans

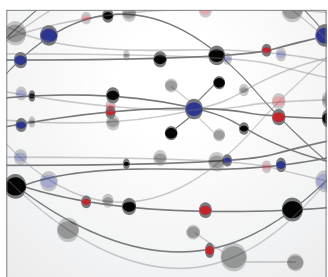

The Scientific World Journal
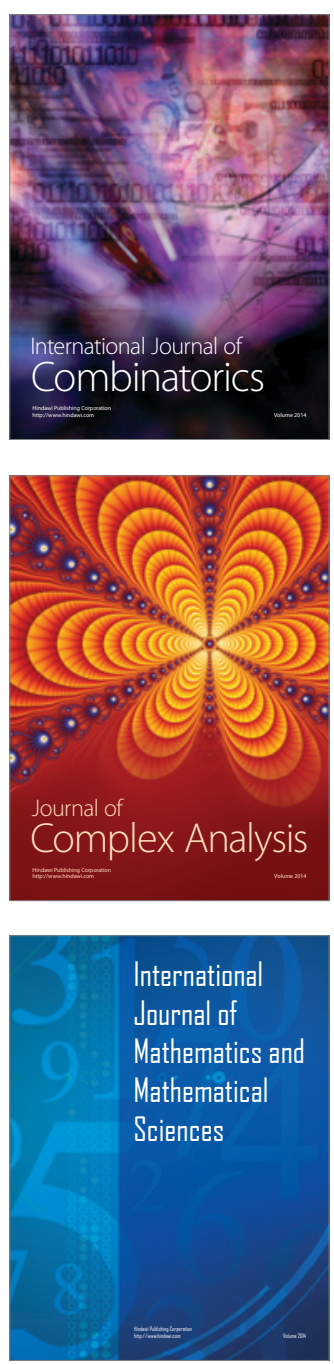
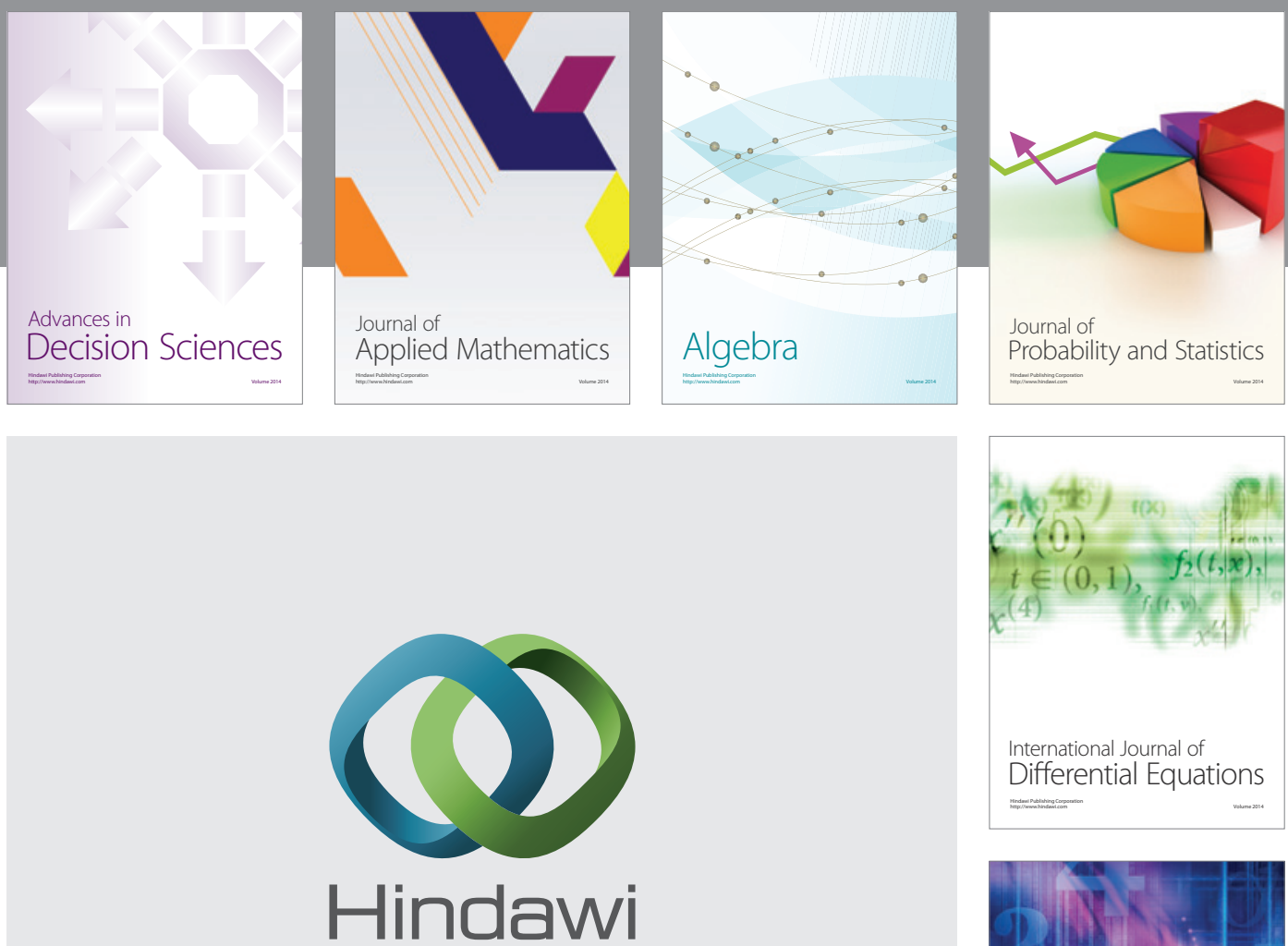

Submit your manuscripts at http://www.hindawi.com
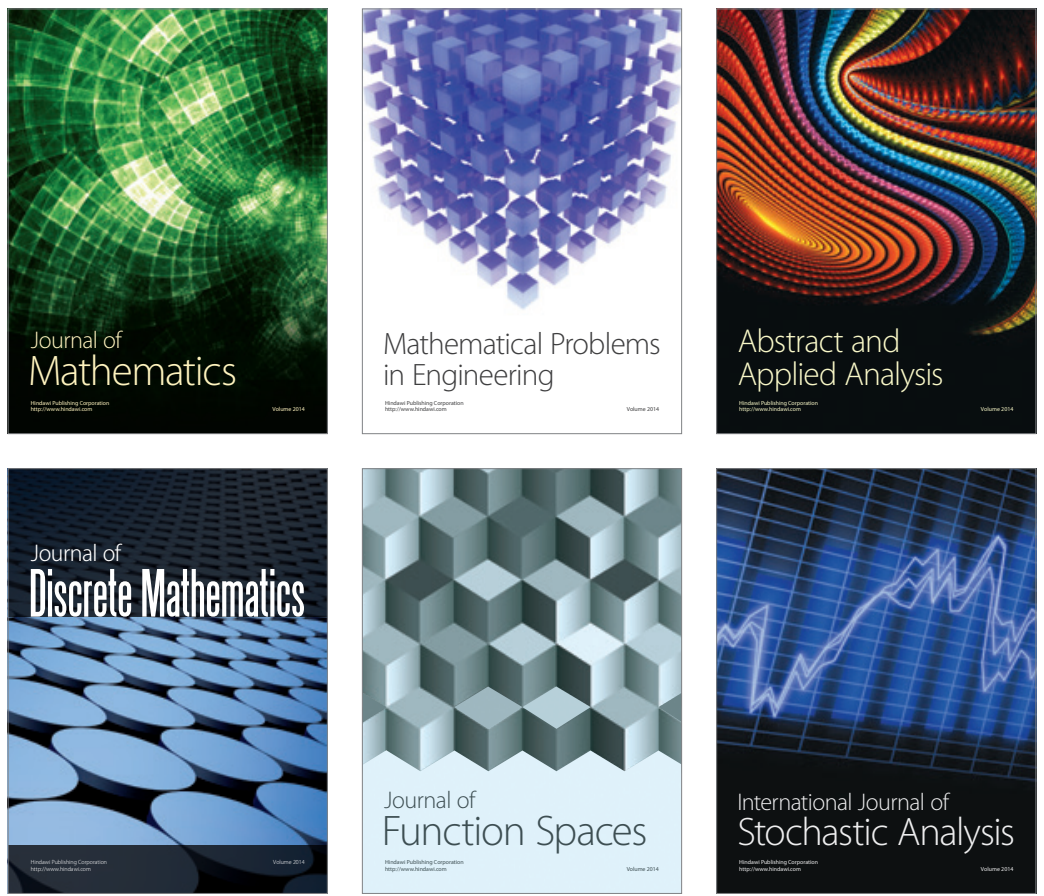

Journal of

Function Spaces

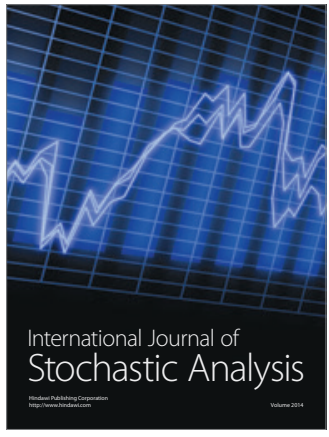

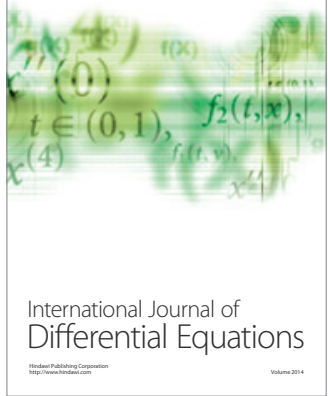
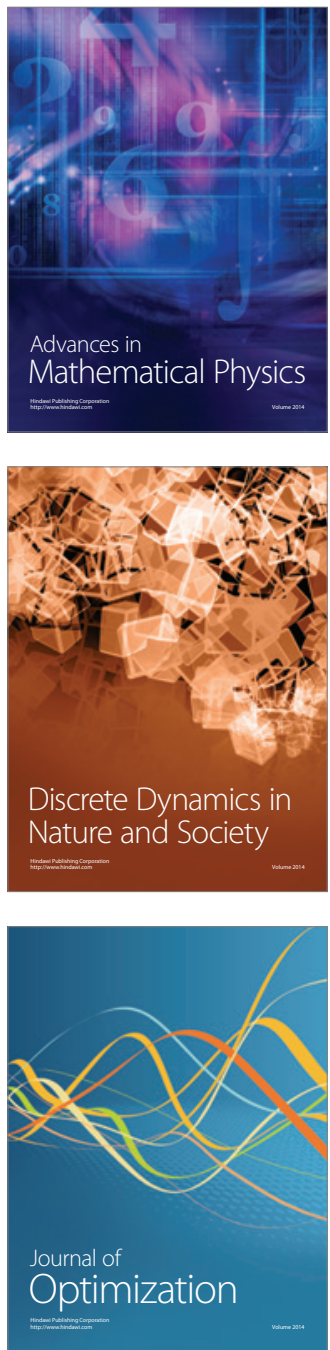